\title{
Une question de principe(s)?
}

\section{Réponse à quelques critiques à l'égard du projet provisoire de «Cadre commun de référence»}

\author{
Matthias E. Storme
}

Published online: 14 August 2008

(C) ERA 2008

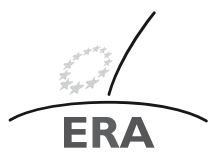

EUROPÄISCHE RECHTSAKADEMIE ACADEMIE DE DROIT EUROPEEN ACADEME DE DROHT EUROPEEN TRIER - TREVES - TREVIR

\section{Introduction}

Le rôle qui m'est ici confié consiste à présenter une réponse à certaines critiques formulées pendant ce Colloque ${ }^{1}$ à l'égard du projet de «cadre commun de référence» $(\mathrm{CCR})^{2}$. Je le ferai dès lors de ma perspective de co-auteur du projet, qui a également été membre de la Commission Lando (Commission on European Contract Law) dès le début de la seconde partie (1992) - mais les réflexions qui suivent n'engagent évidemment que moi-même.

Il s'agit tout d'abord des critiques formulées par les secrétaires généraux de l'Association Henri Capitant pour la culture juridique française (ci-après AHC) et de la Société de législation comparée (ci-après SLC) lors de ce Colloque de Trèves ${ }^{3}$ ou se trouvant dans les travaux de ces Associations sur le projet $\mathrm{CCR}^{4}$. Il s'agit

\footnotetext{
1) Ainsi, je ne mentionne qu'en marge les critiques de Eidenmüller/Faust/Grigoleit/Jansen/Wagner/Zimmermann [6] et dont je n'avais pas connaissance lors du Colloque de Trèves du 6 mars 2008.

2) L'interim outline edition en anglais est disponible à divers sites, dont http://www.storme.be/DCFR interim.html.

3) Denis Mazeaud, «La terminologie commune et les principes directeurs du droit des contrats» et Bénédicte Fauvarque-Cosson, «Les travaux du groupe Association H. Capitant des Amis de la Culture Juridique Française/Société de législation comparée: terminologie, principes directeurs et révision des Principes du droit européen du contrat», également dans ce $\mathrm{n}^{\circ} \mathrm{du}$ ERA-Forum.

4) Publiés en 2 volumes: Association H. Capitant et Société de législation comparée [1] et Association $H$. Capitant et Société de législation comparée [2]. Une version anglaise sera publié chez Sellier sous le titre European Contract Law: Materials for the CFR. Terminology, Guiding principles, Evaluation of PECL.
}

Matthias E. Storme $(\bowtie)$

Professeur à l'Katholieke Universitiet Leuven

Zuidbroek 49, 9030 Gent, Belgique

e-mail: matthias@storme.be

Cet article a été présenté lors d'un séminaire de 1'ERA portant sur «Le Projet de Cadre Commun de Référence», organisé en coopération avec le Joint Network on European Private Law les 6 et 7 mars 2008 à Trèves. L'auteur est Professeur de droit privé et comparé à la K. U.Leuven et l'Universiteit Antwerpen. 
aussi des critiques formulées par le Professeur R. Schulze ${ }^{5}$ et/ou le Professeur Th. Wilhelmsson ${ }^{6}$.

\section{La philosophie}

Nos collègues français ont souligné à raison que, afin d'évaluer un projet, et surtout un projet comme celui du «cadre commun de référence pour un droit privé européen», il faut essayer de saisir ce qu'ils appellent sa philosophie. De même, lorsqu'on compare les caractéristiques de ce projet avec celles d'autres projets ou œuvres, il faut également comparer leur «philosophie» respective. Dans les travaux de nos collègues, certaines critiques de notre projet ont été déduites d'une comparaison avec les Principes Unidroit pour les contrats commerciaux internationaux. Bien que les règles du projet de CCR et les Principes Unidroit aient beaucoup en commun, il y a des différences qui s'expliquent précisément par la différence de «philosophie» de ces deux projets. De plus, pour évaluer le projet de CCR, il aurait été peut-être encore plus pertinent de le comparer avec les droits nationaux et l'acquis du droit communautaire, droits positifs dont la fonction est peut-être plus proche de celle du projet de CCR.

En effet, lorsqu' on compare le projet de CCR et les Principes Unidroit, il faut rester conscient du fait que les Principes Unidroit et le projet de CCR ont un autre but et une autre audience ${ }^{7}$ et que ses auteurs ont une autre responsabilité.

A l'origine des Principes Unidroit se trouvait le vœu d'élaborer et codifier les règles les plus importantes qui régissent déjà en pratique les grands contrats commerciaux internationaux pour des parties contractantes qui veulent échapper aux droits nationaux. Leur but n'est pas d'harmoniser ou d'améliorer les droits nationaux, mais d'y échapper. Ce sont des règles destinées à être appliquées par des arbitres dans les grands arbitrages internationaux. Il s'agit dans ce sens d'un droit très exclusif.

La tâche des auteurs du projet de CCR était au contraire de développer un droit modèle pour les droits nationaux et communautaire, pour tous les contrats, pour tous les citoyens et entreprises, en bref un droit inclusif.

L'origine du projet de CCR est tout autre que l'origine des Principes Unidroit. Ce projet trouve notamment son origine dans la critique de l'acquis communautaire: son pointillisme ${ }^{8}$, sa fragmentarité, son incohérence. Il trouve son origine également dans l'analyse des problèmes de transposition de directives communautaires dans le droit national et des problèmes causés par cette transplantation d'organes communautaires dans les corps de droit nationaux. Ce but exigeait l'étude des domaines avoisinants le droit des contrats, comme le droit de la responsabilité extracontractuelle, de l'enrichissement sans cause, du transfert de propriété mobilière et des sûretés mobilières. Cela résultait d'une étude demandée par la DG SANCO de la

\footnotetext{
5) Présentées d'abord à un Colloque à Munster en décembre 2007 et publiées sous le titre "The Academic Draft of the CFR and the EC Contract Law", voir Schulze [25]. Une réponse à cette critique a déjà été donnée par le prof. Schulte-Nölke [23].

6) Schulze/Wilhelmsson [24].

7) Comp. Bonell [5].

8) I. a. Roth [21]. Voy. déjà en 1981 Kötz [10]; Kötz [11; Hommelhoff [7].
} 
Commission Européenne ${ }^{9}$ et faisait dès lors aussi partie de la tâche confiée au réseau CoPECL ${ }^{10}$.

Il s'agit en plus du vœu d'améliorer et harmoniser les droits nationaux des pays d'Europe, notamment en leur donnant un modèle qui est le fruit d'une science juridique commune.

Il s'agit donc aussi et peut-être même en premier lieu de la revitalisation d'une science juridique européenne comme elle a existé pendant des siècles, une science qui était commune malgré l'absence d'un vrai droit commun ${ }^{11}$, ou plutôt une science qui développait une couche commune, un niveau commun auquel les mille et un droits nationaux, régionaux et locaux (iura propria, ius particulare) participaient. Depuis plus d'un siècle, dans les grandes facultés de droit américaines, on n'enseigne pas d'abord les droits de Connecticut ou Massachusetts, de New York, Illinois ou Californie; on y enseigne une science juridique commune sur le fond duquel les droits étatiques deviennent des variations ${ }^{12}$. Cela devrait être possible en Europe également.

J'étais quand même étonné d'entendre critiquer le projet de CCR parce que les auteurs auraient mis à côté l'expérience nationale ou régionale, surtout en comparant les sources des travaux de nos collègues français et les sources du projet de CCR. Ces travaux sont basés sur une comparaison avec des instruments internationaux, surtout les principes Unidroit, et quelques systèmes ou projets nationaux, surtout français. Les Principes Unidroit à leur tour ne citent que des sources non nationales, ce qui symbolise bien le rejet du droit national. Le projet de CCR au contraire a essayé de synthétiser toute l'expérience des 29 systèmes juridiques de l'Union européenne, 28 droits nationaux ${ }^{13}$ ainsi que l'acquis communautaire, et même celles de quelques pays non membres. Quelques centaines de chercheurs venant de tous les pays de l'UE ont participé ${ }^{14}$. Dans notre entreprise, il n'y a pas de petits pays, et le droit slovène ou estonien est une expérience à prendre au sérieux tout comme le droit anglais, français ou suédois. J'ose même dire que les codes civils de l'Estonie et de la Slovénie sont en matière de droit des obligations pour le moment probablement les meilleurs modèles en droit positif. Un projet pour un droit européen

\footnotetext{
9) C. von Bar \& U. Drobnig, Property Law and Non-contractual Liability Law as they relate to Contract Law, SANCO B5-1000/02/000574, http://ec.europa.eu/comm/consumers/cons_int/safe_shop/fair_bus_ pract/cont_law/study.pdf.

${ }^{10)}$ Les différentes critiques quant au domaine du CCR partent d'ailleurs de perspectives opposées: les critiques de l'AHC/SLC partent d'une comparaison avec des projets internationaux qui par leur nature ne pouvaient comprendre que le droit des contrats; la critique de $R$. Schulze est basée sur l'idée que dans les autres domaines du droit des obligations il n'y aurait pas assez d'acquis communautaire. Il propose de travailler plutôt dans le domaine du droit des sociétés, des marchés de capitaux et des droits intellectuels; tout cela serait bien utile, mais dans une perspective de droit commun (et non seulement droit communautaire) il est plus important d'avoir une bonne base en droit des obligations.

11) Voy. le livre désormais classique de Bellomo [3], en traduction anglaise Bellomo [4].

${ }^{12)}$ L'idée d'enseigner une science juridique commune était le noyau de la grande réforme du Harvard Law School par le doyen C.C. Langdell à partir de 1870.

${ }^{13)}$ En tenant compte de la spécificité du droit écossais notamment, sans exclure les spécificités d'autres droits régionaux.

14) Ayant participé au travaux de la Commission-Lando, du Groupe Acquis et du Study Group, je ne comprends dès lors pas comment le prof. Schulze peut écrire que le DCFR (et dès lors entre autres les travaux du Study Group) est "hardly to claim to be based to an equal extent upon extensive comparative studies and a broad discussion such as the PECL" (in Schulze [25], p. 16).
} 
en matière de contrats ou obligations ne peut dès lors se passer d'une étude de ces droits. Les règles alternatives proposées par l'AHC/SLC comme «PECL révisés» ne se réfèrent ni à l'acquis communautaire ni à la plupart des droits nationaux des pays membres de l'UE. Quant aux critiques du Professeur Schulze, elles attachent une trop grande importance aux fragments d'acquis communautaire et ne prennent dès lors pas au sérieux non plus les expériences des droits nationaux. En paraphrasant, je dirai que développer aujourd'hui les droits nationaux en Europe sans tenir compte d'une perspective européenne ou de droit commun témoigne d'aveuglement, mais se concentrer uniquement sur les projets internationaux et/ou l'acquis communautaire sans prendre au sérieux toute la richesse des droits nationaux témoigne d'une certaine vanité.

Parmi les auteurs et collaborateurs du projet de CCR, il y a certainement de grandes divergences quant aux idées politiques et institutionnelles en matière de droit européen. Mais il y a une foi commune, une foi dans un patrimoine juridique commun des nations européennes. C'est un patrimoine dont les juristes sont les gardiens, non pas comme des conservateurs de musée, mais comme des héritiers responsables de transmettre à leur tour ce patrimoine en le développant, non seulement des héritiers ou légataires mais aussi des trustees et testateurs pour les générations suivantes.

Lorsqu'on discute la philosophie du projet, il faut comprendre cette perspective du projet de CCR et les différences entre cette perspective et celle par exemple des Principes Unidroit.

\section{Les règles et leur structure}

Les AHC et SLC ont publié un modèle alternatif de droit des contrats sous la dénomination révision des PECL. En regardant le contenu et les sources indiqués, il s'agit surtout d'une tentative de fusionner les PECL et le droit français. Evidemment, c'est un exercice légitime, comme il serait aussi légitime de formuler et publier des tentatives de fusionner les PECL ou le projet CCR et le droit allemand, suédois, néerlandais ou hongrois. Mais, comme je l'ai déjà indiqué, ceci n'est pas la perspective du CCR qui a essayé de synthétiser toute l'expérience des 29 systèmes juridiques de l'Union européenne. Ensuite, chacun a évidemment la liberté de se baser dans cet exercice sur les PECL sans tenir compte de tout le travail fait par le Study Group et le Groupe Acquis pendant ces dernières années. Mais une telle approche invite évidemment à une comparaison critique des deux approches et de leurs résultats.

Ce qui m'a par contre étonné, c'est l'idée que le projet de révision des PECL de l'AHC/SCL serait resté plus fidèle au PECL que le projet CCR. Si l'on regarde les textes, et notamment les règles (qui en forment le corps), on voit bien que les règles des livres I à III du CCR - qui couvrent le même domaine que les PECL - sont globalement plus proches des PECL (et dès lors aussi des Principes Unidroit qui y ressemblent) que ne le sont les règles du PECL révisé du projet $\mathrm{AHC} / \mathrm{SLC}$.

Certaines modifications aux PECL dans le projet CCR ont d'ailleurs été inspirées spécifiquement pas les Principes Unidroit, comme par exemple les nouvelles règles en matière de stipulation pour autrui (Art. II-9:301 à 9:303 CCR) ou en matière de correction par le débiteur défaillant («cure», Art. III-3:202 à 204, également inspirées par l'acquis communautaire). 
Notre collègue Fauvarque-Cosson nous dit que le groupe AHC/SLC est resté beaucoup plus fidèle à la structure des PECL: cette affirmation est associé à une critique de la structure du projet de CCR, et notamment la division entre le Livre II («Contrats et autres actes juridiques») et le Livre III («Obligations»). Cela aurait bouleversé la structure des PECL. Une affirmation similaire se trouve dans les critiques de Schulze/ Wilhelmsson.

J'y réponds, parce que cette opposition entre le projet CCR et les PECL est erronée. Si l'on regarde l'ordre des chapitres des Livres I à III, on voit une très grande continuité entre les deux projets. A part les nouveaux chapitres rendus nécessaires par la volonté d'intégrer au lieu de nier le droit positif communautaire, il n'y a presque pas de différence: les Chapitres 1 à 6 des PECL se retrouvent dans le même ordre au projet CCR (sauf la division de l'ancien chapitre 1 en deux), suivi d'un nouveau chapitre contenant des dispositions générales du Livre III et intégrant le chapitre des PECL sur les conditions), ainsi que les Chapitres 7 et suivants du PECL dans le même ordre.

C'est tout autre chose qu'un bouleversement. Le projet de CCR n'a que clarifié la structure en groupant les chapitres en 3 livres, un regroupement qui était nécessaire vu l'importance des matériaux provenant de l'acquis communautaire. Le chapitre 1 des PECL, qui traitait de matières très disparates, a été scindé de sorte que les règles qui concernent les contrats se trouvent dans le Livre II et celles qui concernent le mode d'opérer des règles en général se trouvent au Livre Premier.

C'est également une erreur de dire que le projet CCR aurait procédé à une généralisation beaucoup plus grande que les PECL en adoptant cette «nouvelle» structure. Comme dans les PECL, les règles du Livre II ont été formulées d'abord en termes de contrats; accessoirement, ces règles sont appliquées mutatis mutandis aux actes juridiques unilatéraux. Mais cela était déjà le cas dans les PECL (art. 1:107). A part les règles concernant l'exécution et l'inexécution (PECL Chapitres 7 à 9), les règles des PECL qui se retrouvent dans le Livre III (notamment les Chapitres 10 à 16) étaient déjà, dès leur origine, conçues en termes de droit des obligations et pour être appliquées aux obligations en général ${ }^{15}$. Sous cet aspect, il n'y a que les règles concernant l'exécution et l'inexécution qui ont été «généralisées» en ce sens qu'elles s'appliquent désormais également à l'exécution et l'inexécution des obligations noncontractuelles traitées par le projet CCR (obligations de réparation provenant d'actes illicites, obligation de restitution en cas d'enrichissement sans cause, obligations qui résultent d'une gestion d'affaires). C'était la seule solution raisonnable: nous avons d'abord essayé l'autre solution en rédigeant un chapitre séparé sur l'exécution et l'inexécution des obligations non-contractuelles. Les différences étaient si minces qu'un traitement séparé ne se justifiait pas. Et pour arriver à ce système «unitaire» on n'a guère changé les règles sur l'exécution et l'inexécution des obligations contractuelles; au contraire, à quelques exceptions près (changement de circonstances, résolution, etc.), elles étaient (et sont) valables pour les obligations non-contractuelles.

Il est exact que les Principes Lando étaient non seulement dans leurs solutions comme le projet CCR - mais également dans leur structure proches des Principes Unidroit. Mais cela s'explique facilement. La Commission Lando était une entreprise

\footnotetext{
${ }^{15)}$ Ils semblent d'ailleurs bien aptes pour un droit autonome des contrats également, puisque les chapitres
} correspondants des Principes Unidroit diffèrent guère de celles des PECL. 
pionnière (commencée en 1980 !), en petit comité, qui a fait un travail remarquable dans un domaine plus restreint. Le Study Group a pris sa responsabilité pour le droit des contrats vu dans le contexte du droit privé entier, et donc dans ses liens avec les autres branches du droit des obligations et certaines parties du droit des biens. C'est un choix qui a d'ailleurs été proposé par une réponse commune de la Commission Lando et du Study Group à la Communication de la Commission européenne concernant le droit des contrats de juillet $2001^{16}$. Il est clair qu'un instrument qui ne devient applicable que par le choix des parties, ne peut avoir qu'un domaine plus restreint qu'un instrument qui s'adresse au législateur, et peut dès lors envisager le tout sous l'angle du contrat (ou de l'acte juridique) seulement.

Si l'on revient dans le monde du droit positif, du droit national et communautaire, même en restant fidèles aux Principes Lando, la structure du projet CCR est plus justifiée que celle des Principes Unidroit. La plupart des droits nationaux de l'Europe connaissent bien la notion d'un droit des obligations dont le droit des contrats fait partie et ont un régime unitaire pour l'exécution et l'inexécution des obligations. Dans un projet de droit commun, il ne fait pas de sens de revenir sur ces acquis du développement juridique. Ils connaissent également la notion d'acte juridique et les traitent de manière similaire au projet CCR. Le Code national qui a intégré le plus l'expérience des PECL est le Code civil estonien: mais la structure du Code civil estonien est proche du projet CCR (et pour éviter tout malentendu: le Code civil estonien précède le projet $\mathrm{CCR}$ ): le Code civil estonien contient une partie générale qui correspond largement aux Livres I et II du CCR et un livre sur le droit des obligations qui correspond largement au Livres III à VII du CCR. Il est vrai que la structure du Code civil estonien est également proche de celle du Code civil allemand. Et alors? Si l'on trouve dans le Code civil allemand, dans cet ordre, des règles concernant les personnes, les actes juridiques, les obligations, les biens, la famille et les successions, cela correspond à la nature des choses: les gens naissent, s'engagent, acquièrent de la propriété, se marient, ont des enfants et meurent. Il y a d'ailleurs des différences importantes entre la structure du projet CCR et le Code civil allemand. Ainsi, dans ce dernier, les règles de formation des contrats ne se trouvent pas dans la partie générale. Dans le projet CCR, toutes les règles concernant les formation des contrats au sens large (formation, validité, représentation) se trouvent dans le même Livre. Le Livre II est beaucoup moins abstrait que la partie générale du Code civil allemand: il ne concerne pas tout les actes juridiques, mais uniquement celle du droit des obligations et des biens.

Il est exact que le CCR a pris soin de distinguer le contrat comme acte juridique de la relation contractuelle qui se compose de droits et obligations contractuelles. Mais est-ce une généralisation? Une super-abstraction? Il me semble qu'en distinguant deux choses dans la notion ambigüe de contrat, on a précisé au lieu de généralisé.

Cette précision constitue d'ailleurs un progrès remarquable. Toute une série de règles ont pu être formulées de manière plus claire et précise, comme par exemple celles sur les effets de la résolution d'un contrat. La distinction a une importance particulière dans les relations multipartites où il faut absolument distinguer entre les actes juridiques (contrats) et les relations obligationnelles qui ne continuent pas né-

\footnotetext{
16) Octobre 2001, http://www.sgecc.net/pages/en/home/137.joint_response_to_the_eu_commissions_communication_on_european_contract_law.htm.
} 
cessairement entre les parties au contrat. Ainsi, la relation entre le cessionnaire d'une obligations contractuelle et le débiteur cédé est bien une relation contractuelle, bien qu'il n'y ait pas de contrat entre eux. En voulant absolument enserrer le droit des obligation dans le contrat, le projet alternatif risque de créer pas mal d'ambigüités et d'erreurs.

Bien sûr, le projet CCR n'est pas parfait. Mais on ne peut le juger que de manière relative, en le comparant à des instruments alternatifs. Il ne devrait pas être trop difficile de trouver des améliorations ponctuelles. Les travaux de 1'AHC/SDL contiennent d'ailleurs toute une série de suggestions intéressantes ${ }^{17}$. Certaines ont déjà été reprises par le projet CCR (modifications en 2008), notamment quant au terme ou à la durée du contrat (prolongation tacite), quant au comportement inconciliable (estoppel) ou la position dominante comme élément d'interprétation défavorable dans les contrats.

Mais il faut constater que les critiques adressées au contenu du projet CCR sont loin d'être unanimes et souvent en sens opposées. Ainsi, certains auteurs ou groupes critiquent le projet comme étant néolibéral (je me demande toujours ce que ceux qui emploient ce mot comprennent sous ce terme qui aujourd'hui constitue surtout un slogan), généralement sans que cette critique soit basée sur une analyse du texte comme il est. (Un procès d'intention est évidemment plus facile). D'autres critiquent le projet comme portant une atteinte trop grande à la liberté contractuelle (critique généralement plus précise que la première $)^{18}$. La critique de l'AHC/SDL est moins unilatérale mais aussi plus ambigüe: d'une part le projet est critiqué parce que l'on aurait restreint le rôle à jouer par les exigences de la bonne foi ${ }^{19}$, d'autre part l'alternative ne tiendrait pas compte de l'acquis communautaire, notamment en matière de droit de la consommation et ignore les formes contemporaines de la protection du consommateur. D'une part, le projet CCR est critiqué parce que les règles seraient trop abstraites, d'autre part les exemples concrèts à l'appui de cette critique ${ }^{20}$ (découpage du principe de bonne foi en plusieurs règles, réglementation trop détaillée des devoirs d'information précontractuelle) critiquent le manque de généralité ${ }^{21}$. Le Professeur Schulze, pour sa part, critique le fait que les règles concernant les obligations contractuelles ont été extrapolées aux obligations non contractuelles, mais son alternative consiste à transformer la plupart des règles pointillistes de l'acquis communautaires dans des matières généralement très spécifiques - ce ne sont toujours que «des îles dans une mer de droit national» ${ }^{22}$ - en règles générales pour tous les contrats (en particulier les règles concernant l'inexécution de la Directive Garanties en matière de biens de consommation $^{23}$ ), une «politique de généralisation» qui a été critiquée a juste titre par d'autres auteurs ${ }^{24}$. La critique de Monsieur Schulze est d'autant plus ambigüe en ce

\footnotetext{
17) Dont certaines ont d'ailleurs été reprises par le projet CCR (modifications en 2008), p.ex. quant au terme ou durée du contrat.

${ }^{18)}$ P.ex. Eidenmüller/Faust/Grigoleit/Jansen/Wagner/Zimmermann [6], p. 537 suiv.

${ }^{19)}$ Critique formulée en se référant à un article de Lando [12].

${ }^{20)}$ Dans la contribution du prof. B. Fauvarque-Cosson.

${ }^{21)}$ Je voudrais quand-même remarquer que le projet alternatif de PECL revisé a d'autre part des règles beaucoup plus détaillées que le projet CCR en d'autres matières, p.ex. les conditions ou le terme (durée) des obligations.

22) Expression de Rittner [20].

23) Proposée par Schulze [25], p. 12.

24) Jansen/Zimmermann [8], également en anglais Jansen/Zimmermann [9].
} 
que, d'une part, il critique le projet CCR parce qu'il s'alignerait trop exclusivement sur les PECL (notamment dans le livre III) ${ }^{25}$, mais d'autre part propose de remplacer le projet CCR par une compilation des PECL et des ACQP (Acquis Principes) ... ${ }^{26}$ Tout cela ne veut pas dire que les critiques et suggestions concrètes concernant les règles spécifiques ne sont pas parfois justifiées et presque toujours utiles au débat. Je voudrais seulement dire que ces critiques concrètes sont plus utiles que des considérations générales et abstraites critiquant la philosophie ou la structure sans «coller» au texte même du projet CCR.

Quant à la critique concrète à l'égard de la reformulation de la règle de la bonne foi, je voudrais rappeler d'abord que d'autres ont critiqué le projet CCR pour des raisons diamétralement opposées, notamment parce qu'il donnerait une place trop grande au principe de bonne foi ... En tout cas, la reformulation ne concerne pas tant le domaine d'application de cette règle (et les devoirs qui en découlent), mais les effets spécifiques d'une violation de la bonne foi, les «remedies». Dans le projet CCR, le bonne foi est toujours source de devoirs et de déchéances, mais pas d'obligations autonomes au sens plein du terme.

\section{Les principes}

Enfin, je voudrais répondre à certains critiques qui concernent le manque de «principes» et plus spécialement d'un énoncé de «principes directeurs» en forme de règles au Livre Premier.

Le mot principe est malheureusement usé et abusé dans beaucoup de sens. Afin de savoir dans quelle forme et place devraient se trouver des principes dans un projet CCR, il faut d'abord clarifier de quoi on parle.

Dans un premier sens, on emploie le mot «principe» dans le sens de «règle» distinguée de «l'exception»: lex specialis derogat generali. Ici il s'agit d'une règle, qui s'applique à défaut de règles plus spécifiques. Le projet de CCR comme toute œuvre de législation, contient beaucoup de ces règles. Dans un certain sens, toute règle de droit est potentiellement un principe dans ce sens.

Parfois on parle de principe dans le sens inverse: une règle ayant valeur constitutionnelle et dès lors ayant priorité sur des règles contraires n'ayant pas cette valeur.

Enfin, il y a les «principes sous-jacents», pour employer l'expression de PECL et le projet CCR. Ce sont des valeurs sous-jacentes ayant valeur explicative et interprétative. Le projet CCR a corrigé ici une incohérence terminologique des PECL. Les PECL employaient le mot principe aussi bien pour les règles, qui s'appelaient principes, que pour les principes sous-jacents à ces règles. Un tel principe est une règle dont manquent les conditions d'application et qui dès lors ne doit pas être appliqué sans que ce principe soit concrétisé dans des règles (au sens plus strict du mot).

\footnotetext{
${ }^{25)}$ Critique formulée par Schulze [25], p. 12.

26) Proposition développée plus en détail par Schulze/Wilhelmsson [24].
} 
Dans une certaine tradition française à laquelle se réfèrent nos collègues français ${ }^{27}$, on trouve une approche de ces principes qui diffère clairement de l'approche de beaucoup d'autres traditions de droit national sous deux aspects.

Dans le projet CCR, les principes sont contradictoires ou plutôt en conflit. Elles expriment les valeurs en conflit, entre lesquelles le législateur ou autre juriste qui développe le droit doit trouver un équilibre dans des problèmes et cas concrets. Un tel principe n'est pas limité par une ou plusieurs exceptions, mais par d'autres principes qui ont également une valeur plus ou moins générale.

Dans cette approche française, les principes sont plutôt des règles assorties d'exceptions exprimés ailleurs. A mon avis, cette dernière approche est une approche trompeuse du système juridique. Ainsi, les règles de protection du consommateur ne sont pas seulement des exceptions au principe de la liberté contractuelle, mais des règles qui expriment d'autres valeurs que les valeurs au fond de la liberté contractuelle. Ce n'est pas au niveau des principes eux-mêmes que ce conflit peut être tranché. Ce conflit est tranché par des règles concrètes qui essayent de trouver un équilibre entre les valeurs en conflit, qui précisent les conditions d'application dans lesquelles l'une ou l'autre valeur a la priorité.

Deuxièmement, dans le projet $\mathrm{CCR}$, ces principes se trouvent en dehors des règles, ne sont pas des règles et ne peuvent dès lors être appliqués en tant que tel. Dans l'approche française, les principes ont l'ambition d'être des règles applicables à défaut d'exception. Mais en y regardant mieux, ils ont la forme d'une «synecdoque», comme l'ont décrit nos collègues italiens Sacco et Monateri ${ }^{28}$. Ainsi, les concepts de «principe» et de «définition» ont dans la tradition française une autre signification que dans la tradition anglaise, allemande ou néerlandaise et la plupart des autres droits nationaux.

On nous dit que ces principes expriment les idées majeures, les grandes idées du système juridique. Cela implique que les autres aspects des règles juridiques ne seraient pas importants, seraient de seconde catégorie. Cela donne même l'impression qu'il y a des grands juristes qui s'occupent des grandes idées qui forment le ciel juridique, et des petits juristes qui s'occupent de toutes ces questions techniques terre-à-terre.

Certainement, les grandes idées ont une place dans le discours juridique, mais seulement lorsqu'on se rend compte du caractère contradictoire de ces idées et de l'importance de tout le travail ingrat de formuler des règles précises qui servent à un système de droit équilibré et forment des instruments directement utiles pour résoudre des cas concrets.

Quelle est alors la place des grands principes en droit privé? Prenons le droit des contrats. Mon livre modèle, qui m'a été donné comme exemple par mon directeur de thèse, l'ancien Avocat Général à la Cour de Justice des Communautés européennes Walter van Gerven, est la thèse de doctorat du Professeur J.H. Nieuwenhuis: Drie beginselen van contractenrecht (Trois principes du droit des contrats) ${ }^{29}$. Il y démontre de manière brillante comment une grande partie du droit des contrats peut-être com-

\footnotetext{
27) L'exemple de droit positif généralement cité est celui du Nouveau Code de procédure civile français, qui contient un chapitre de "Principes directeurs". A ma connaissance, aucun code civil a suivi ce modèle.

${ }^{28)}$ Sacco [22], p. 15; Monateri [15]; Monateri [16].

29) Publié chez Kluwer, Deventer 1979.
} 
prise comme un équilibre entre trois principes: le principe d'autonomie, le principe de la confiance et le principe de réciprocité. Chacun de ces trois principes n'est vrai que tempéré par les deux autres principes; dans l'absolu ils sont contradictoires et en conflit. Dans ce sens, ces principes n'ont qu'une valeur relative et non apodictique. Il est même dangereux d'élever ces principes en règles sans les «couper». ${ }^{30} \mathrm{Si}$ vraiment, on veut à côté des règles précises, introduire des principes, ils ne peuvent dès lors pas prendre la forme de synecdoques, de règles incomplètes, mais seulement une forme relative, ou plutôt «comparative». Ainsi, plus l'autonomie d'une partie est réelle, moins le juge peut intervenir pour contrebalancer un déséquilibre entre les obligations.

La notion de propositions comparatives comme éléments du système juridique a été analysée surtout par des auteurs autrichiens et allemands comme Wilburg ou $\mathrm{Otte}^{31}$. Mais ces auteurs n'ont jamais envisagé d'introduire ces principes dans un Code comme règles légales. Ils les voyaient comme des recommandations au législateur et surtout comme une œuvre de doctrine et non de législation.

Je crois en effet qu'il ne faut pas mélanger les genres.

C'est la tâche du législateur de produire de bonnes règles, inspirées par des valeurs, essayant d'équilibrer les valeurs en cause, mais non pas de faire de la philosophie. Dans beaucoup de droits nationaux ainsi que dans le droit communautaire, il y a déjà trop d'éléments rhétoriques, précatives et/ou philosophiques dans les règles ${ }^{32}$.

C'est une tâche de la science juridique, de la doctrine, d'expliciter les valeurs en cause et les conflits de valeurs et d'apporter les interprétations des règles en tenant compte de ces conflits de valeurs et de leur évolution ${ }^{33}$.

C'est la tâche des juges de tenir compte de ces interprétations et des circonstances de la cause pour décider les cas concrets en litige.

En conséquence, les règles du projet CCR ne contiennent pas des principes sous la forme française. L'énoncé des principes sous-jacents se fait non sous forme d'articles mais dans un commentaire introductif en forme d'essai, qui comprend une liste tentative des principes ou valeurs sous-jacentes. Notre collègue Fauvarque-Cosson a pu écrire que «L'équilibre entre tous ces principes et leurs correctifs» (dans cette introduction) «est subtil» et que dès lors, sans une formulation précise des principes dans la forme que l'AHC/SLC a produit, «la plus grande insécurité règnera». Eidenmüller/e.a./Zimmermann ont également écrit que manquent des «règles de conflit» qui clarifieraient dans quelles conditions un principe aurait priorité sur un autre ${ }^{34}$.

Mais la formulation «précise» dont on a besoin pour éviter la plus grande insécurité doit se trouver non pas dans des «principes» en forme de synecdoque, mais dans les règles, règles qui précisément devraient exprimer l'équilibre choisi par l'auteur

\footnotetext{
${ }^{30)}$ Expression de Nieuwenhuis [17], p. 505, https://openaccess.leidenuniv.nl/bitstream/1887/3186/1/ 353 003.pdf.

31) Wilburg [27]; Wilburg [26]; Otte [18]; Otte [19].

${ }^{32)}$ Pour une analyse de certains de ces éléments, voy. entre autres l'article de Sacco [22].

${ }^{33)}$ Comp. Luhmann [14], traduit en espagnol et en italien.

34) Eidenmüller/Faust/Grigoleit/Jansen/Wagner/Zimmermann [6], p. 535.
} 
de ces règles. Les principes eux-mêmes sont incapables de décider l'équilibre entre eux $^{35}$; les règles de conflit demandées ne sont autres que les règles elles-mêmes.

Evidemment, tout travail de doctrine qui essaie d'expliciter ces principes sous forme de propositions, commentaires et notes, est utile; il est même indispensable qu'une telle littérature doctrinale se développe. Mais cela reste une œuvre de doctrine, qui n'a ni la même fonction ni la même autorité qu'une œuvre de législation. Il serait même dangereux et créateur de grande insécurité d'introduire ces principes dans le texte même des articles. Demandez à des associations de juristes d'autres pays que la France de faire le même travail, et il en résulterait une synthèse très différente. Cela n'est pas un drame, c'est précisément preuve de la richesse de nos traditions nationales.

Prenons concrètement les principes recommandés par le travail de l'AHC/SLC. De ma perspective, non pas comme co-auteur de l'œuvre législative, mais comme auteur de doctrine, j'y trouve à boire et à manger. Mais je trouve également que ces principes ne reflètent pas l'ensemble des valeurs sous-jacentes. Je trouve même qu'en les insérant dans le corps du CCR, on y introduirait toute une série d'ambigüités et de contradictions avec les (autres) règles du CCR.

J'en donne ici quelques exemples.

Il est à mon avis contre-productif de formuler un principe général de bonne foi (proposition 0:302) sans préciser les sanctions ou remèdes d'une violation des exigences de la bonne foi. C'est ce que le projet CCR a fait dans des articles spécifiques comme l'art. III-1:103.

On créerait à mon avis une grande insécurité en insérant un principe comme «Le contrat ne peut produire effet qu'autant qu'il ne réalise pas une atteinte ou modification illégitime aux droits des tiers» (proposition 0:102, al. 2). Le projet CCR a des règles plus précises pour différentes types de violations.

Le principe proposé au 0:103 «La révocation unilatérale n'est efficace qu'en matière de contrats à durée indéterminée» est d'abord incomplet et deuxièmement formulé de manière beaucoup moins précise que l'article III-1:109 CCR. Quel est le sens d'élever une seule de ces règles en principe et de le reformuler de manière incomplète?

La même remarque vaut pour le principe $\mathrm{n}^{\circ} 0: 201$, al. 3: «En cours d'exécution, la force obligatoire du contrat peut être remise en cause si un changement imprévisible de circonstances compromet gravement l'utilité du contrat pour l'une des parties». C'est une des modifications possibles des obligations des parties, que l'on trouve de manière plus précise à l'article III-1:110.

De nouveau la même remarque pour le principe 0:202: «Chacune des parties peut exiger de son cocontractant l'exécution de son obligation telle qu'elle a été prévue par le contrat». On trouve une règle plus précise aux articles III-1:110 (1) (devoir d'exécuter) et III-3:301 et 302 (concernant le droit de faire exécuter en nature). Quelle est la valeur ajoutée d'un principe qui donne les parties un droit à l'exécution lorsqu'il y a des règles précises indiquant les circonstances dans lesquelles il y a ou il n'y a pas un droit d'exiger l'exécution en nature?

\footnotetext{
35) Comp. Luhmann [13], également dans in Soziologische Aufklärung, spéc. p. 189 suiv., traduit en espagnol et en italien.
} 
Ainsi, le principe proposé au n ${ }^{\circ}$ 0:203 selon lequel «Le contrat crée une situation que les tiers doivent respecter et dont ils peuvent se prévaloir sans pouvoir en poursuivre l'exécution» mélange différentes questions complexes dans une formule ambigüe. D'une part, ce principe impose aux tiers un devoir de non-ingérence dans des relations contractuelles. Cette règle est trop générale: sous certaines conditions, une ingérence constituera un acte illicite, sanctionnée par les règles de la responsabilité extra-contractuelle. La règle précise se trouve dans le CCR à l'art. VI-2:211. La deuxième partie de la phrase est en partie une question de droit des biens. Je sais que dans la doctrine française, ce principe est invoqué pour expliquer l'effet erga omnes du transfert de propriété en vertu d'un contrat (plus correctement d'une obligation contractuelle). Cela devrait être réglementée de manière plus précise dans le droit des biens, qui détermine dans quelles conditions les tiers doivent accepter et respecter ce transfert de propriété.

\section{Conclusion}

Le projet de CCR fait partie d'un débat en cours. Comme membre des groupes qui ont rédigé les textes, je ne peux qu'être satisfait de l'attention que donnent des collègues partout en Europe aux résultats intermédiaires du projet et des analyses critiques qu'ils font. Certaines critiques ont déjà donné lieu à des modifications du projet. Dans d'autres cas, nous avons au moins le devoir de répondre pourquoi elles ne sont pas suivies. C'est ce que j'ai essayé de faire dans cet article.

\section{Références}

1. Association H. Capitant et Société de législation comparée: Projet de cadre commun de référence. Terminologie contractuelle commune, coll. Droit privé européen et comparé, 6 (2008)

2. Association H. Capitant et Société de législation comparée: Projet de cadre commun de référence. Principes contractuels communs, coll. Droit privé européen et comparé, 7 (2008)

3. Bellomo, M.: L’Europa del diritto comune (1988), 7ième éd., Rome (1994)

4. Bellomo, M.: The common legal past of Europe 1000-1800, Washington (1998)

5. Bonell, J.: The UNIDROIT Principles of International Commercial Contracts and the Principles of European Contract Law: Similar Rules for the Same Purposes? Uniform Law Review 26, 229 suiv. (1996)

6. Eidenmüller, H., Faust, F., Grigoleit, H.C., Jansen, N., Wagner, G., Zimmermann, R.: Der gemeinsame Referenzrahmen für das europäische Privatrecht. JZ, p. 529 suiv. (2008)

7. Hommelhoff, P.: Zivilrecht unter dem Einfluss europäischer Rechtsangleichung, 192. AcP, p. 71 suiv. (1992)

8. Jansen, N., Zimmermann, R.: Grundregeln des bestehenden Gemeinschaftsprivatrechts? JZ, p. 113 suiv. (2007)

9. Jansen, N., Zimmermann, R.: Restating the Acquis communautaire? A Critical Examination of the "Principles of the Existing EC Contact Law", in MLR (2008)

10. Kötz, H.: Gemeineuropäisches Privatrecht, in: Festschrift für Konrad Zweigert, Mohr Tübingen, p. 481 suiv. (1981)

11. Kötz, H.: Rechtsvereinheitlichung - Nutzen, Kosten, Methoden, Ziele, 50. RabelsZ 1986, p. 5 suiv.

12. Lando, O.: The Structure and the Legal Values of the Common Frame of Reference (CFR). ERCL 3, 245 suiv. (2007)

13. Luhmann, N.: Positives Recht und Ideologie, 53. Archiv für Rechts- und Sozialphilosophie (1967), également dans Soziologische Aufklärung, vol. I, Westdeutscher Verlag, Köln-Opladen (1970)

14. Luhmann, N.: Rechtssystem und Rechtsdogmatik, Kohlhammer, Stuttgart (1974)

15. Monateri, P. G.: La sineddoche, Milano (1984) 
16. Monateri, P. G.: Règles et techniques de la définition en France et en Allemagne. RIDC, p. 77 suiv. (1984)

17. Nieuwenhuis, J.H.: Legitimatie en heuristiek van het rechterlijk oordeel. Rechtsgeleerd magazijn Themis, p. 494 suiv. (1976)

18. Otte, G.: Komparative Sätze im Recht, Zur Logik eines beweglichen Systems, 2. Jahrbuch für Rechtssoziologie und Rechtstheorie, Bertelsmann, Düsseldorf (1972)

19. Otte, G.: Zur Anwendung komparativer Sätze im Recht, Das bewegliche System im geltenden und künftigen Recht, Wilburg Symposium 1985, Springer, Wien (1986)

20. Rittner, F.: Das Gemeinschaftsprivatrecht und die europäische Integration. JZ, p. 851 suiv. (1995)

21. Roth, W.-H.: Transposing pointillist EC guidelines into Systematic National Codes - Problems and Consequences. ERPL, p. 761 siuv. (2002)

22. Sacco, R.: Legal formants: a Dynamic Approach to Comparative Law. American Journal of Comparative Law 39, p. 1 suiv. (1991)

23. Schulte-Nölke, H.: Contract Law or Law of Obligations? The Draft Common Frame of reference (DCFR) as a multifunctional tool, dans: Common Frame of Reference and Existing EC Contract Law, ed. R. Schulze, Sellier, p. 47 suiv. (2008)

24. Schulze, R., Wilhelmsson, Th.: From the Draft Common Frame of Reference towards European Contract Rules. ERCL, p. 154 suiv. (2008)

25. Schulze, R.: The Academic Draft of the CFR and the EC Contract Law, dans: Common Frame of Reference and Existing EC Contract Law, ed. R. Schulze, Sellier, p. 3 suiv. (2008)

26. Wilburg, W.: Elemente des Schadensrechts, Marburg (1941)

27. Wilburg, W.: Zusammenspiel der Kräfte im Aufbau des Schuldrechts. AcP, 379 suiv. (1964) 